\title{
Pushing the limits of techniques in small-sample biopsy of lung adenocarcinoma
}

\author{
Chuong D. Hoang, MD \\ From the Section of Thoracic Surgery, National Institutes of Health, National Cancer Institute, Center for Cancer \\ Research and Clinical Center, Bethesda, Md \\ Disclosures: Author has nothing to disclose with regard to commercial support. \\ Received for publication Feb 25, 2017; accepted for publication March 3, 2017; available ahead of print April 1, \\ 2017. \\ Address for reprints: Chuong D. Hoang, MD, Thoracic and Gastrointestinal Oncology Branch, Section of \\ Thoracic Surgery, National Institutes of Health, National Cancer Institute, Center for Cancer Research and \\ Clinical Center, 10 Center Dr, Rm 4-3940, Mail code 1201, Bethesda, MD 20892 (E-mail: chuong.hoang@ \\ nih.gov). \\ J Thorac Cardiovasc Surg 2017; 154:340-1 \\ $0022-5223 / \$ 0.00$ \\ Published by Elsevier Inc. on behalf of The American Association for Thoracic Surgery \\ http://dx.doi.org/10.1016/j.jtcvs.2017.03.013
}

Our recent increased understanding of lung adenocarcinoma pathobiology culminated in a new classification system summarized by the International Association for the Study of Lung Cancer. ${ }^{1}$ Before this paradigm, adenocarcinoma represented a widely divergent clinical, radiologic, molecular, and pathologic spectrum with a variable prognosis that is poorly predicted by the older classification systems. Now, adenocarcinoma is better characterized by preinvasive, minimally invasive, and invasive histologic subtypes as described semiquantitatively in 5\% tumor increments according to formalized guidelines for assigning a predominant pattern. There is consensus that histologic subtypes associate with clinical outcome, wherein for invasive adenocarcinoma the lepidic pattern has a favorable prognosis, papillary and acinar patterns have an intermediate prognosis, and micropapillary/ solid patterns have the worst prognosis. 2,3

Huang and colleagues ${ }^{4}$ explore an intriguing notion to employ preoperative nonsurgical biopsy techniques to determine whether adenocarcinoma subtyping is accurate or not. They present 128 patients who had preoperative biopsy and lung surgery for invasive adenocarcinoma. They assessed the accuracy of preoperative computed tomography-guided core needle or radial probe endobronchial ultrasound biopsy results versus the pathologic specimen. However, the authors only achieved a concordance rate of $58.6 \%$ (75 out of 128) in the predominant histologic pattern among preoperative biopsy and surgical specimen. This lack of accuracy led to their main realization that the complex histologic features of lung adenocarcinoma preclude reliable assessment by small-sample biopsy techniques.

Preoperative clinical implications of this result are multiple (assuming that frozen section analysis remains an imperfect method). Without accurate and reliable preoperative subtyping, it is more difficult to plan for a limited resection versus completion lobectomy. Because some adenocarcinoma subtypes may be associated with nodal

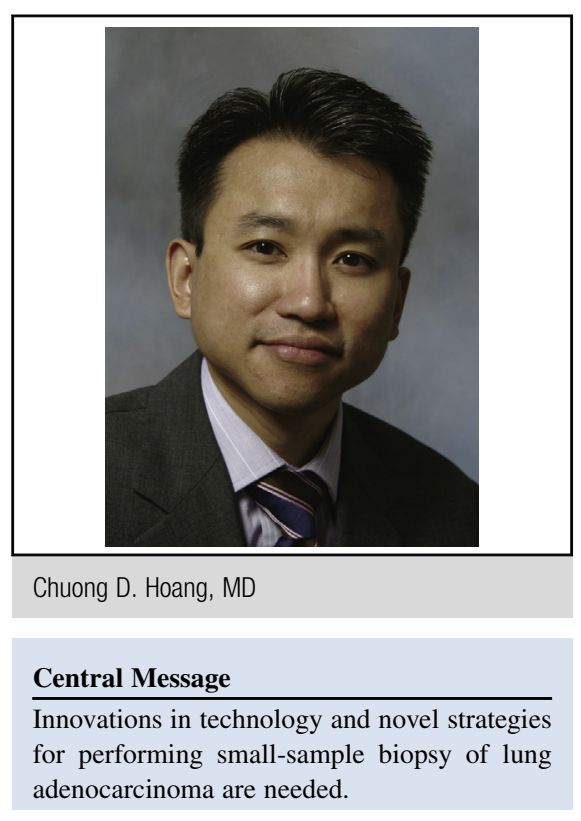

See Article page 332

metastases, ${ }^{5}$ this preoperative information could determine the need and extent of lymph node dissection. Other possible scenarios relate to those patients who cannot tolerate lung surgery. What next-best therapy is available for certain adenocarcinoma subtypes in lieu of lung resection? Finally, we can extrapolate to ask whether accurate histologic subtyping could determine which early stage patients may benefit from neoadjuvant therapy.

It is instructive to understand the technical limitations likely responsible for the overall results of this study. As the authors indicated, computed tomography-guided core needle cannot always traverse the entire tumor diameter due to needle trajectory or intervening structures (eg, vasculature and airways). Tissue volume retrieved in the core needle is fixed, so the extent of representative sampling is limited and proportionally smaller as tumor mass increases. Multiple needle passes via different trajectories are not unlimited to allow access in all tumor regions to overcome sampling bias (secondary to inherent intratumor cellular heterogeneity ${ }^{1}$ ). Radial probe endobronchial ultrasound biopsy relies on transbronchial biopsy using a forceps $(\sim$ $1.5 \mathrm{~mm}$ ), often augmented with cytologic brushings; alternatively, a $21 \mathrm{G}$ needle can be used. ${ }^{6}$ The ultrasound probe is positioned in an airway with an endobronchial tumor or in an adjacent airway (necessitating needle aspiration). 
Extensive sampling of tumor regions is not possible with biopsy forceps, for this would require burrowing into or through the entire tumor. These limited degree(s) of freedom for biopsy approach might be solved with future innovation in hardware and other emerging biopsy modalities.

A valuable point is reaffirmed by this study in that histologic subtyping of adenocarcinoma influences prognosis.

\section{References}

1. Travis WD, Brambilla E, Noguchi M, Nicholson AG, Geisinger KR, Yatabe Y, et al. International Association for the Study Of Lung Cancer/American Thoracic Society/European Respiratory Society International Multidisciplinary Classification of Lung Adenocarcinoma. J Thorac Oncol. 2011;6:244-85.
2. Hung JJ, Yeh YC, Jeng WJ, Wu KJ, Huang BS, Wu YC, et al. Predictive value of the International Association for the Study of Lung Cancer/American Thoracic Society/European Respiratory Society classification of lung adenocarcinoma in tumor recurrence and patient survival. J Clin Oncol. 2014;32:2357-64.

3. Warth A, Muley T, Meister M, Stenzinger A, Thomas M, Schirmacher P, et al. The novel histologic International Association for the Study of Lung Cancer/American Thoracic Society/European Respiratory Society classification system of lung adenocarcinoma is a stage-independent predictor of survival. J Clin Oncol. 2012;30:1438-46

4. Huang K-Y, Ko P-Z, Yao C-W, Hsu C-N, Fang H-Y, Tu C-Y, et al. Inaccuracy of lung adenocarcinoma subtyping using preoperative biopsy specimens. J Thorac Cardiovasc Surg. 2017;154:332-9.e1.

5. Xu L, Tavora F, Burke A. Histologic features associated with metastatic potential in invasive adenocarcinomas of the lung. Am J Surg Pathol. 2013;37:1100-8.

6. Chen A, Chenna P, Loiselle A, Massoni J, Mayse M, Misselhorn D. Radial probe endobronchial ultrasound for peripheral pulmonary lesions. A 5-year institutional experience. Ann Am Thorac Soc. 2014;11:578-82. 